\title{
ELECTRON ATTRACTION AS A MECHANISM FOR THE CHEMICAL BOND OF GROUND STATE $\mathrm{H}_{2}$ *
}

by

Donald Greenspan

Mathematics Department

University of Texas at Arlington

$T R \# 294$

\section{ABSTRACT}

Previously, electron attraction, incorporated into classical models of the chemical bond, yielded correct bond lengths and vibrational frequencies for all the diatomic molecules through $\mathrm{O}_{2}$. In each case, maximally symmetric electron-nuclei configurations were utilized. In this paper, which concentrates only on ground state $H_{2}$, it is shown that maximal symmetry is not necessary for the attainment of correct results.

* Calculations performed on the Cray YMP at the University of Texas Center for High Performance Computation 
1. Introduction. Theoretical work has shown that pairs of electrons need not always repel $(1,2)$. Nobelist John Bardeen and his associates showed, for example, that the quantum mechanical theory for superconductivity requires electron attraction (1). This is not inconsistent with the recent work on electron substructure by Nobelist H. G. Dehmelt $(3,4)$. Motivated by these current ideas, we have been exploring the possibility that the chemical bond is the result of electron attraction. The resulting models are semiclassical in the sense that they incorporate a nonstandard force into Newtonian dynamical equations. We have shown ${ }^{(5-7)}$ that classical dynamical simulation with paired electron attractions yields the correct bond lengths and vibrational frequencies for the diatomic molecules $\mathrm{H}_{2}^{1}, \mathrm{H}_{2}^{2}, \mathrm{H}_{2}^{3}, \mathrm{H}^{1} \mathrm{H}^{2}, \mathrm{H}^{1} \mathrm{H}^{3}, \mathrm{Li}_{2}{ }^{7}, \mathrm{~B}_{2}{ }^{11}, \mathrm{C}_{2}{ }^{11}, \mathrm{~N}_{2}{ }^{14}, \mathrm{O}_{2}{ }^{16}, \mathrm{Li}^{7} \mathrm{H}^{1}$ and $\mathrm{Li}^{7} \mathrm{H}^{2}$. In each case, a maximum of symmetry was incorporated into the calculations. In this paper, by concentrating only on ground state $\mathrm{H}_{2}$, we will show that a maximum of symmetry is not necessary to approximate correctly the experimental bond length and experimental vibrational frequency, which are, respectively, $0.742 \AA$ and $(1.318) 10^{14}$ H $(8,9)$

2. Mathematical Preliminaries. Note first that the energy $E$ of ground state $\mathrm{H}_{2}$ is $(10)$

$$
E=-(50.7289) 10^{-12} \text { erg. }
$$

Next, let us denote the electrons by $P_{1}$ and $P_{3}$, while the protons are denoted by $\mathrm{P}_{2}$ and $\mathrm{P}_{4}$. Assume for the moment that the only forces are coulombic. In cgs units, for $i=1,2,3,4$, and at any time $t$, let $P_{i}$ be located at $\vec{r}_{i}=\left(x_{i}, y_{i}, z_{i}\right)$, have velocity $\vec{v}_{i}=\left(\dot{x}_{i}, \dot{y}_{i}, \dot{z}_{i}\right)$, and have acceleration $\vec{a}_{i}=\left(\ddot{x}_{i}, \ddot{y}_{i}, \ddot{z}_{i}\right)$. Then the classical equations of motion for the $P_{i}$ are 
$(2.2)$

$$
m_{i} \vec{a}_{i}=\sum_{\substack{j=1 \\ j \neq i}}^{4} \frac{e_{i} e_{j}}{r_{i j}} \frac{\vec{r}_{j i}}{r_{i j}}, i=1,2,3,4,
$$

in which $\vec{r}_{j i}$ is the vector from $P_{j}$ to $P_{i}, r_{i j}=\left\|\vec{r}_{i j}\right\|$, and

$$
e_{1}=e_{3}=-e_{2}=-e_{4}=(-4.8028) 10^{-10} \text { esu, }
$$

$$
\mathrm{m}_{1}=\mathrm{m}_{3}=(9.1085) 10^{-28} \mathrm{~g} \text {, }
$$

$$
\mathrm{m}_{2}=\mathrm{m}_{4}=(16724) 10^{-28} \mathrm{~g} \text {. }
$$

We assume now that $P_{1}$ and $P_{3}$ attract, rather than repel. For computational convenience, we also set $\vec{R}_{i}=\left(X_{i}, Y_{i}, Z_{i}\right)$ and make the transformations

$$
\vec{R}_{i}=10^{12} \vec{r}_{i}
$$

$$
\mathrm{T}=10^{22} \mathrm{t} \text {. }
$$

Then system (2.2) transforms into the following system:

$$
\frac{d^{2} X_{1}}{d T^{2}}=(2.5324576)\left(-\frac{x_{1}-X_{2}}{R_{12}{ }^{3}}-\frac{x_{1}-X_{3}}{R_{13}{ }^{3}}-\frac{X_{1}-X_{4}}{R_{14}}\right)
$$

$$
\frac{\mathrm{d}^{2} Y_{1}}{d T^{2}}=(2.53245 .6)\left(-\frac{Y_{1}-Y_{2}}{R_{12}{ }^{3}}-\frac{Y_{2}-Y_{3}}{R_{13}{ }^{3}}-\frac{Y_{1}-Y_{4}}{R_{14}{ }^{3}}\right)
$$

$$
\frac{d^{2} Z_{1}}{d T^{2}}=(2.5324576)\left(-\frac{Z_{1}-Z_{2}}{R_{12}{ }^{3}}-\frac{Z_{1}-Z_{3}}{R_{13}{ }^{3}}-\frac{Z_{1}-Z_{4}}{R_{14}{ }^{3}}\right)
$$

(2.11) $\frac{d^{2} X_{2}}{d T^{2}}=(1.379269) 10^{-3}\left(-\frac{X_{2}-X_{4}}{R_{12}{ }^{3}}-\frac{X_{2}-X_{3}}{R_{23}{ }^{3}}+\frac{X_{2}-X_{4}}{R_{24}{ }^{3}}\right)$

(2.12) $\quad \frac{d^{2} Y_{2}}{d T^{2}}=(1.379269) 10^{-3}\left(-\frac{Y_{2}-Y_{1}}{R_{12}{ }^{3}}-\frac{Y_{2}-Y_{3}}{R_{23}{ }^{3}}+\frac{Y_{2}-Y_{4}}{R_{24}{ }^{3}}\right)$

(2.13) $\quad \frac{d^{2} Z_{2}}{d T^{2}}=(1.379269) 10^{-3}\left(-\frac{Z_{2}-Z_{1}}{R_{12}{ }^{3}}-\frac{Z_{2}-Z_{3}}{R_{23}{ }^{3}}+\frac{Z_{2}-Z_{4}}{R_{24}{ }^{3}}\right)$ 


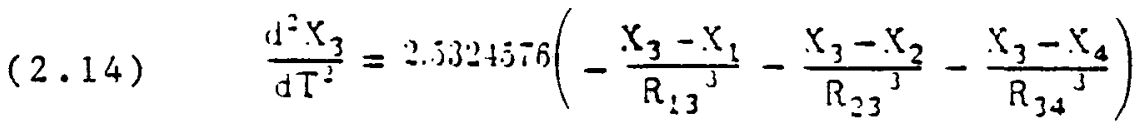

(2.15) $\quad \frac{\mathrm{d}^{2} Y_{3}}{\mathrm{~d} T^{2}}=2.3324576\left(-\frac{Y_{3}-Y_{1}}{R_{13}{ }^{3}}-\frac{Y_{3}-Y_{2}}{R_{23}{ }^{3}}-\frac{Y_{3}-Y_{4}}{R_{34}{ }^{3}}\right)$

(2.16) $\quad \frac{d^{2} Z_{3}}{d T^{2}}=2.5324576\left(-\frac{Z_{3}-Z_{1}}{R_{13}{ }^{3}}-\frac{Z_{3}-Z_{2}}{R_{23}{ }^{3}}-\frac{Z_{3}-Z_{4}}{R_{34}{ }^{3}}\right)$

(2.17) $\frac{d^{2} X_{4}}{d T^{2}}=(1.379269) 10^{-3}\left(-\frac{X_{4}-X_{1}}{R_{14}{ }^{3}}+\frac{X_{4}-X_{2}}{R_{24}{ }^{3}}-\frac{X_{4}-X_{3}}{R_{34}{ }^{3}}\right)$

(2.18) $\frac{d^{2} Y_{4}}{d T^{2}}=(1.379269) 10^{-3}\left(-\frac{Y_{4}-Y_{1}}{R_{24}{ }^{3}}+\frac{Y_{4}-Y_{3}}{R_{24}{ }^{3}}-\frac{Y_{4}-Y_{3}}{R_{34}{ }^{3}}\right)$

(2.19) $\quad \frac{\mathrm{d}^{2} Z_{4}}{\mathrm{dT}^{2}}=(1.379269) 10^{-3}\left(-\frac{Z_{4}-Z_{4}}{R_{14}{ }^{3}}+\frac{Z_{4}-Z_{3}}{R_{24}{ }^{3}}-\frac{Z_{4}-Z_{3}}{R_{34}{ }^{3}}\right)$

It is system (2.8)-(2.19) which we will solve numerically from arbitrarily selected initial data. However, conservation of energy is essential in the numerical procedure, so that we must and will utilize numerical methodology designed for just such a purpose (11).

Note that for convenience we set

$$
\vec{v}_{i}=\left(\frac{d X_{i}}{d T}, \frac{d Y_{i}}{d T}, \frac{d Z_{i}}{d T}\right)
$$

and observe that

$$
\vec{v}_{i}=10^{10} \vec{v}_{i}
$$

Finally; observe that the total energy $E$ of the system is given by 
$(2.20)$

$$
\begin{aligned}
E & =\frac{1}{2}(9.1085) 10^{-28}\left(\mathrm{v}_{1}{ }^{2}+\mathrm{v}_{3}{ }^{2}\right)+\frac{1}{2}(16724) 10^{-28}\left(\mathrm{v}_{2}{ }^{2}+\mathrm{v}_{4}{ }^{2}\right) \\
& +(23.06689) 10^{-20}\left(-1 / \mathrm{r}_{12}-1 / \mathrm{r}_{13^{-1 / r_{14}}}-1 / \mathrm{r}_{23}+1 / \mathrm{r}_{24}-1 / \mathrm{r}_{34}\right),
\end{aligned}
$$

or, equivalently, by

$$
\begin{aligned}
& E=\frac{1}{2}(9.1085) 10^{-8}\left(\mathrm{~V}_{1}{ }^{2}+\mathrm{V}_{3}{ }^{2}\right)+\frac{1}{2}(16724) 10^{-8}\left(\mathrm{~V}_{2}{ }^{2}+\mathrm{V}_{4}{ }^{2}\right) \\
& +(23.06689) 10^{-8}\left(-1 / \mathrm{R}_{12^{-1 / R_{13}}}{ }^{-1 / \mathrm{R}_{14}}-1 / \mathrm{R}_{23}+1 / \mathrm{R}_{24}-1 / \mathrm{R}_{34}\right) .
\end{aligned}
$$

3. Maximally Symmetric systems. In setting initial data for computer calculations, we first incorporate as much symetry as possible and repeat, but with increased accuracy, calculations reported previously $(5)$. Thus, we set $P_{1}$ at $(0,0, Z), P_{2}$ at $(X, 0,0)$, $\mathrm{P}_{3}$ at $(0,0,-\mathrm{Z})$, and $\mathrm{P}_{4}$ at $(-\mathrm{X}, 0,0)$. The velocities of $\mathrm{P}_{2}$ and $\mathrm{P}_{4}$ are taken to be zero, initially, while the velocities of $P_{1}$ and $P_{3}$ are taken to be $(0, V Y, 0)$ and $(0,-V Y, 0)$, respectively. The value VY will be determined from (2.1) and (2.21) after all other initial data are given. Calculations were made with several choices of $\triangle T$ to assure accuracy. The results to be reported are for $\Delta T=2.0$. The implicit convergence tolerance for position was $10^{-6}$, while that for velocity was $10^{-13}$. Simulations were executed for 40,000,000 time steps. The energy was conserved throughout each calculation. A typical vector computer program is available 12 for the interested reader. The results are given in Table I.

Since the average experimental frequency $f$ is $(1.318) 10^{14} \mathrm{H}$ and the average experimental molecular diameter $d$ is $0.742 \AA$, it is then seen readily that all the results in Table I are in good agreement with experiment. This is unexpected because the choices of initial data may be inconsistent in that, for each given set of initial positions, the velocities of $P_{2}$ and $P_{4}$ need not necessarily be 
equal to zero. Moreover, though initial data can be contrived readily to yield $f$ or $d$ to within acceptable bounds, initial data cannot be chosen to assure, a priori, the correctness of both $f$ and $d$.

TABLE I

\begin{tabular}{|l|l|l|l|l|l|}
\hline & $\mathrm{X}$ & $\mathrm{Z}$ & $\mathrm{VY}$ & $\mathrm{f}\left(10^{14} \mathrm{H}\right)$ & $\mathrm{d}(\mathrm{A})$ \\
\hline 1 & 4000 & 4500 & 0.033020594 & 1.366 & 0.776 \\
3 & 4000 & 4200 & 0.034271092 & 1.375 & 0.770 \\
4 & 4000 & 4000 & 0.035125168 & 1.383 & 0.764 \\
5 & 3800 & 5000 & 0.031242354 & 1.377 & 0.794 \\
6 & 4435 & 5000 & 0.030437537 & 1.363 & 0.790 \\
7 & 3000 & 4360 & 0.035006180 & 1.339 & 0.808 \\
\hline
\end{tabular}

4. Partially symmetric systems. In section 3, all systems considered yielded two dimensional motion for $P_{1}$ and $P_{3}$ in the $Y Z$ plane, symmetrically about the origin, and yielded one dimensional motion for $\mathrm{P}_{2}$ and $\mathrm{P}_{4}$ along the $\mathrm{X}$ axis, symmetrically about the origin. In this section we consider sets of initial data which yield fully three dimensional motions under a modicum of symmetry. In all the cases to be considered the initial values of $Y_{1}, Y_{2}, Z_{2}, Y_{3}, Y_{4}$, and $Z_{4}$ are zero. Thus, the electrons and the protons, initially, are in the $X Z$ plane. The initial velocity vectors of $P_{2}$ and $P_{4}$ are taken to be zero. The initial velocity vector for $\mathrm{P}_{1}$ is $(0, V Y, 0)$ and that for $P_{3}$ is $(0,-V Y, 0)$. The remaining initial positions are given in Table II. The value VY in the table was determined from (2.1) and $(2.21)$. The time step was $\Delta T=2.0$ and the results were checked periodically with $\Delta T=0.2$. The tabular selections were motivated by the better results in Table I. Each case was run for a minimum of $40,000,000$ time steps. 
TABLE II

\begin{tabular}{|l|c|c|c|c|c|c|c|c|c|}
\hline & $\mathrm{X}_{1}$ & $\mathrm{Z}_{1}$ & $\mathrm{X}_{2}$ & $\mathrm{X}_{3}$ & $\mathrm{Z}_{3}$ & $\mathrm{X}_{4}$ & $\mathrm{VY}$ & $\mathrm{d}(\mathrm{A})$ & $\mathrm{E}\left(10^{14} \mathrm{H}\right)$ \\
\hline $\mathrm{I}$ & 1000 & 5000 & 4200 & 1000 & -5000 & -4200 & 0.03082428 & 0.7984 & 1.351 \\
\hline 2 & 1000 & 4000 & 4200 & 1000 & -4000 & -4200 & 0.03491311 & 0.7721 & 1.374 \\
4 & 1000 & 5000 & 3800 & 1000 & -5000 & -3800 & 0.03126779 & 0.8051 & 1.334 \\
5 & 1000 & 3600 & 3800 & 1000 & -3600 & -3800 & 0.03763350 & 0.7662 & 1.362 \\
6 & 1000 & 3800 & 3600 & 1000 & -3800 & -3600 & 0.03706020 & 0.7795 & 1.319 \\
7 & 1000 & 5000 & 3400 & 1000 & -5000 & -3400 & 0.03158437 & 0.8184 & 1.322 \\
8 & 500 & 3600 & 3800 & -500 & -3600 & -3800 & 0.03739651 & 0.7553 & 1.412 \\
9 & 500 & 3800 & 3600 & -500 & -3800 & -3600 & 0.03688544 & 0.766 .5 & 1.392 \\
10 & 500 & 4000 & 4000 & -500 & -4000 & -4000 & 0.03513952 & 0.7663 & 1.393 \\
\hline
\end{tabular}

In each case in Table II, electron motion could be characterized as follows. The motion of $P_{1}$, for example, was that of oscillation from a position near $P_{2}$ to a position near

$\mathrm{P}_{4}$ and then back and forth again. All the tabular results are considered to be good. The results of Case 4 are particularly good in that both the frequency and the diameter differ from the experimental results by $3 \%$. In Case 5 , the diameter is in error by $5 \%$, but the frequency is in error by only $0.08 \%$. Case 6 shows a relatively large diameter but a compensating decrease in frequency. For Table II, the average diameter is $0.7855 \%$ and the average frequency is $(1.354) 10^{14} \mathrm{H}$, which are in error by $5.8 \%$ and $2.7 \%$, respectively.

5. Nonsymmetric Systems. Our initial approach to nonsymmetric systems was as follows. The protons were set on the $x$ axis initially with $3000 \leqslant x_{2} \leqslant 7000$ and with $x_{4}=-x_{2}$. Pairs of typical nonsymmetric initial positions for $P_{1}$ and $P_{3}$ were, for example, 
(a) $\quad(1,0,4000),(0,0,-4000)$

(b) $\quad(1000,0,3800),(0,0,-3800)$

(c) $\quad(5000,0,4000),(0,0,-2500)$

(d) $\quad(3500,0,2000),(-6000,0,-3500)$.

The initial velocities for $P_{2}$ and $P_{4}$ were zero, while those for $P_{1}$ and $\mathrm{P}_{3}$ were $(0, \mathrm{VY}, 0),(0,-V Y, 0)$, respectively. The constant $V Y$ was determined from (2.1) and (2.21). A variable time step was implemented as follows. For $i=1,2,3,4 ; j=1,2,3,4$,

$$
\begin{aligned}
& \Delta T=2.0 \text { if min }\left|P_{i} P_{j}\right|>3000 \\
& \Delta T=0.2 \text { if min }\left|P_{i} P_{j}\right|<3000 \\
& \Delta T=0.02 \text { if min }\left|P_{i} P_{j}\right|<2000 \\
& \Delta T=0.002 \text { if min }\left|P_{i} P_{j}\right|<1000 \\
& \Delta T=0.0002 \text { if min }\left|P_{i} P_{j}\right|<500 .
\end{aligned}
$$

In a11, 74 cases were run. In 72 of these, $\mathrm{P}_{1}$ and $\mathrm{P}_{3}$, in time, orbited each other in a close orbit whose diameter was smaller than 1000 units. In six of these cases, one of $P_{1}$ or $P_{3}$ was "sling shotted" outward with a sufficiently high velocity which made the time steps in use suspect. The resulting nonperiodic motions, the need for small time steps, and the very large variety of possible initial data made the choice of a reasonable sample and of computer implementation unfeasible at the present time. The following simplistic approach, first recommended by Gillespie (13), was therefore implemented.

The closely orbiting electron pair phenomenon was simulated by combining $P_{1}$ and $P_{3}$ into a single particle of charge and mass twice that of a single electron. The new particle was denoted by $P_{1}$ and the protons were now denoted by $P_{2}$ and $P_{3}$. Initialiy, $P_{2}$ was set at $\left(X_{2}, 0,0\right)$ and $P_{3}$ at $\left(-X_{2}, 0,0\right)$ with zero velocities. $P_{1}$ was set at $\left(X_{1}, 0, Z_{1}\right)$ with velocity $(0, V Y, 0)$. The value VY was determined from the energy equation 


$$
\begin{aligned}
-(50.7289) 10^{-4}= & \frac{1}{2}(18.2170)(V Y)^{2} \\
& +23.06689\left(-2 / R_{12}-2 / R_{13}+1 / R_{23}\right) .
\end{aligned}
$$

selected computer results are recorded in Table III. In each case the time step was $\Delta T=2.0$. The vibrational motions were larger than those which resulted in previous sections. The average diameter and average frequency of the tabulated results are $0.7399 \AA$ and $(1.592) 10^{14} \mathrm{H}$, respectively. Considering the simplicity of the model, the results recorded in the table are deemed to be of sufficient accuracy to warrant the more precise, full, four-body study using relatively small time steps.

TABLE III

\begin{tabular}{|l|l|l|l|l|l|l|l|}
\hline & \multicolumn{1}{|c|}{$x_{1}$} & $z_{1}$ & $x_{2}$ & $x_{3}$ & $V Y$ & $d(\AA)$ & $f\left(10^{14}{ }_{H}\right)$ \\
\hline 1 & 0 & 7000 & 5500 & -5500 & 0.018727837 & 0.7384 & 1.634 \\
2 & 0 & 7000 & 6000 & -6000 & 0.018186690 & 0.7637 & 1.613 \\
3 & 500 & 6000 & 4500 & -4500 & 0.022639535 & 0.6984 & 1.667 \\
4 & 500 & 7000 & 5000 & -5000 & 0.019167789 & 0.7258 & 1.603 \\
5 & 500 & 6000 & 6000 & -6000 & 0.020660680 & 0.8300 & 1.408 \\
6 & 1000 & 4000 & 4500 & -4500 & 0.029315004 & 0.6830 & 1.629 \\
\hline
\end{tabular}


References

1. J. Bardeen, L. N. Cooper, and J. R. Schrieffer, Phys. Rev., 108, $1957, \mathrm{p} 1175$.

2. W. M. Honig, D. W. Kraft, and E. Panarella, QUANTUM UNCERTAINTIES, Plenum, N.Y., 1987 , pp 147, 151 .

3. H. Dehmelt, Proc. Nat. Acad. Sci. USA, 86, $1989, \mathrm{p} 8618$.

4. R. F. O'Connell, Found. Phys., 23, 1993, p 461.

5. D. Greenspan, Mo1. Phys., 74, 1991, p 1015.

6. D. Greenspan, Phys. Essays, 5, 1992, p 554.

7. D. Greenspan, Comp. Math. Applic., 25, 1993, p 75.

8. L. Pauling, THE NATURE OF THE CHEMICAL BOND, Corneli Univ: Press, Ithaca, 1973 .

9. B. H. Mahan, UNIVERSITY CHEMISTRY, Addison-Wesley, Reading, MA, 1966.

10. G. Herzberg, MOLECULAR SPECTRA AND MOLECULAR STRUCTURE, van Nostrand, N.Y., 1965, P 532.

11. D. Greenspan, ARITHMETIC APPLIED MATHEMATICS, Pergamon, oxford, 1980, p p 20-30.

12. D. Greenspan, TR \#278, Math: Dept., Univ. Texas at Arlington, 1991.

13. R. J. Gillespie, J. Chem. Ed., 40, 1963, p 295. 